\title{
Enterocistoplastia de ampliación laparoscópica: experiencia inicial
}

\author{
Núñez Mora C, Cansino Alcaide R, Alonso Gregorio S, Martínez-Piñeiro Lorenzo L, \\ De la Peña Barthel J.
}

Servicio de Urología. Hospital Universitario La Paz. Universidad Autónoma de Madrid.

Actas Urol Esp. 2007:31(1):17-22

\section{RESUMEN}

ENTEROCISTOPLASTIA DE AMPLIACIÓN LAPAROSCÓPICA: EXPERIENCIA INICIAL

Objetivo: Analizar la experiencia inicial de nuestro grupo en la realización de enterocistoplastia de ampliación por vía laparoscópica.

Pacientes y métodos: Describimos la técnica de enterocistoplastia de ampliación con segmento ileal realizada completamente mediante abordaje laparoscópico. Presentamos los casos de dos pacientes diagnosticados de vejiga hiperrefléxica refractaria a tratamiento médico que fueron sometidos a esta intervención. En ambos casos la técnica se realizó sin complicaciones intraoperatorias, aunque requirió un tiempo quirúrgico de 6 y 4,5 horas respectivamente. Los resultados con un seguimiento de 12 y 6 meses fueron satisfactorios en ambos, consiguiendo una vejiga de baja presión con una buena continencia.

Conclusiones: La enterocistoplastia de ampliación laparoscópica es una técnica compleja, que requiere una amplia experiencia, sobre todo en sutura laparoscópica. Reproduce absolutamente a la técnica abierta y aporta las ventajas inherentes al abordaje laparoscópico.

Palabras Clave: Enterocistoplastia de ampliación. Laparoscopia. Vejiga neurógena.

\section{ABSTRACT}

\section{LAPAROSCOPIC AUGMENTATION ENTEROCYSTOPLASTY: INICIAL EXPERIENCE}

Objective: To analyze the initial experience of our group in the realization of the augmentation enterocystoplasty by laparoscopyc approach.

Methods and patients: We describe the augmentation enterocystoplasty technique with ileal segment completely achieved by laparoscopyc approach.

We present the cases of two patients suffering from hyperreflexic bladder refractory to medical treatment who underwent this surgery. In both cases the technique was realized without intraoperative complications although it was needed a surgical time of 6 and 4,5 hours respectively. The results after 12 and 5 months were satisfactory in both patients, obtaining a low pressure bladder with a good continence.

Conclusions: Laparoscopyc augmentation enterocystoplasty is a complicated technique that requires a great experience, mainly in laparoscopyc suture. It reproduces completely the open surgery and it offers all the advantages inherent to the laparoscopyc surgery.

Keywords: Augmentation enterocystoplasty, laparoscopy, neurogenic bladder.

$\mathrm{L}$ a enterocistoplastia de ampliación constituye una técnica ampliamente difundida y aceptada para el tratamiento de aquellos pacientes con vejiga neurogénica hiperrefléxica refractaria a tratamientos conservadores ${ }^{1-10}$.
Casi todas las series publicadas presentan los resultados obtenidos mediante cirugía abierta, siendo muy escasos las series en las que la cirugía se realiza asistida por laparoscopia ${ }^{1,11,12}$ y más raras aún las realizadas mediante cirugía laparoscópica pura ${ }^{11}$. 
En este trabajo describimos la técnica utilizada por nosotros para la realización de enterocistoplastia de ampliación mediante abordaje integramente laparoscópico y presentamos los resultados obtenidos en los dos primeros pacientes intervenidos mediante esta técnica.

\section{DESCRIPCIÓN DE LA TÉCNICA}

Realizamos preparación intestinal el día previo a la cirugía mediante solución evacuante $\mathrm{y}$ descontaminación intestinal antibiótica.

Bajo anestesia general procedemos, en posición de litotomía, a cateterización ureteral bilateral con catéteres extracorpóreos que son fijados a una sonda vesical $16 \mathrm{Ch}$.

En decúbito supino y con una posición de Trendelenburg de unos $20^{\circ}$ colocamos, mediante técnica abierta, un trocar supraumbilical de 12 $\mathrm{mm}$. A continuación insertamos otro trocar de 12 $\mathrm{mm}$, otros 2 de $11 \mathrm{~mm}$ y otros 2 de $5 \mathrm{~mm}$, de forma similar a la que utilizamos en la cistectomía radical laparoscópica (Fig. 1).

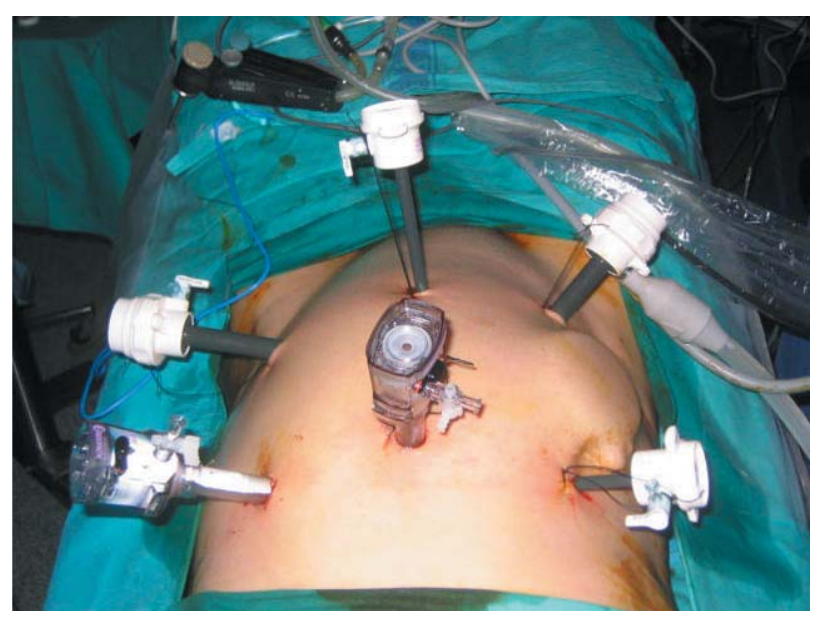

FIGURA 1. Disposición de los trócares.

Tras la movilización del ciego mediante incisión en la línea de Toldt seleccionamos un asa ileal que alcance la pelvis sin tensión. Introducimos una óptica auxiliar por un trocar de 11 $\mathrm{mm}$ procediendo a transiluminar el meso ileal con el fin de identificar las arcadas vasculares (Fig. 2), aislando un asa de entre 15 y $25 \mathrm{~cm}$ de longitud en función de las necesidades de ampliación. Seccionamos el asa y mesos seleccionados mediante endocortadora (Endo-GIA). Con

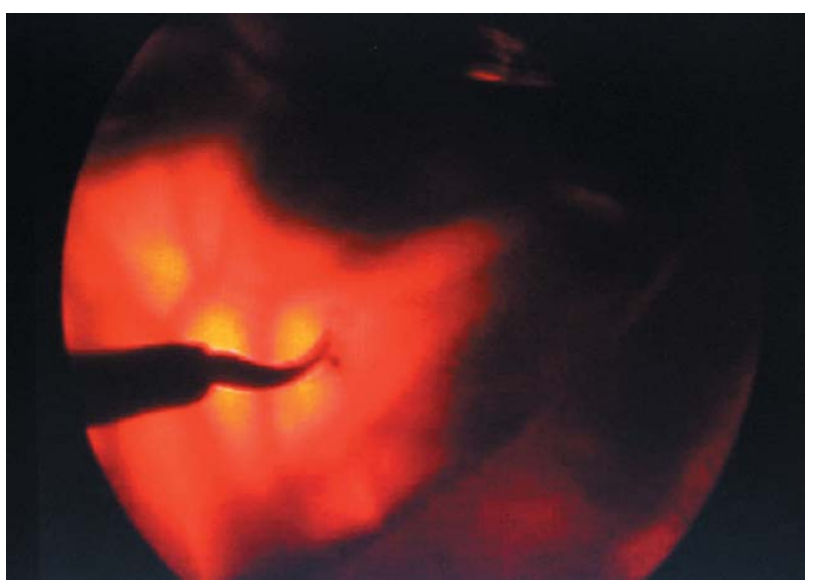

FIGURA 2. Transiluminación del meso intestinal. Elección del segmento ileal conservando las arcadas vasculares.

el fin de restaurar la continuidad intestinal fijamos ambos cabos en posición latero-lateral con 2 puntos de seda. Realizamos una enterotomía en cada cabo por la que introducimos una EndoGIA, comunicando ambos cabos y cerrando a continuación la enterotomía mediante una sutura continua de Monocryl ${ }^{\circledR}$ 3-0. Suturamos la brecha mesentérica con puntos sueltos de seda.

Tras retirar las líneas de grapas de ambos extremos del asa ileal desfuncionalizada destubularizamos ésta por su borde antimesentérico. En el caso de haber seleccionado un asa larga, de unos $25 \mathrm{~cm}$, la plegamos en "U" mediante una sutura continua de Monocryl ${ }^{\circledR}$ 3-0.

Seccionamos las arterias umbilicales, liberando el espacio de Retzius, y realizamos una cistotomía sagital desde el cuello vesical en su cara anterior hasta la barra intertrigonal (Fig. 3).

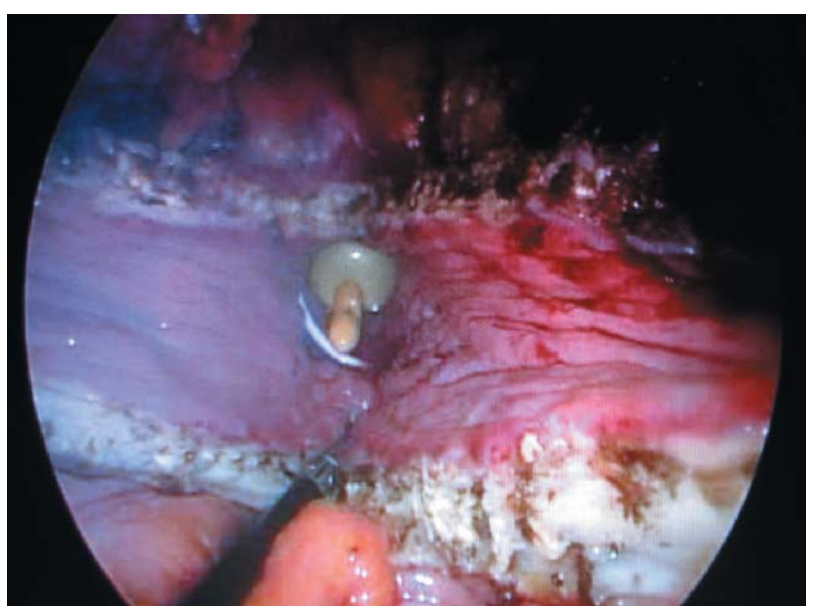

FIGURA 3. Cistotomía sagital desde cuello vesical a las 12 del horario cistoscópico hasta la barra intertrigonal. 
Iniciamos la anastomosis entero-vesical en el extremos inferior de la cistotomía, avanzando por cuadrantes mediante suturas continuas interrumpidas de Monocryl ${ }^{\circledR}$ 3-0. Primero anastomosamos los dos cuadrantes posteriores y a continuación los dos anteriores. Una vez completada la anastomosis comprobamos la estanqueidad de la sutura, reforzando con puntos sueltos si es necesario. Dejamos dos drenajes aspirativos, uno sobre la enterocistoplastia y otro sobre la anastomosis intestinal.

\section{CASOS CLÍNICOS}

\section{Caso 1}

Varón de 23 años de edad diagnosticado de vejiga hiperrefléxica secundaria a mielomeningocele. Portador de derivación ventrículo-peritoneal por hidrocefalia. A pesar del tratamiento médico el paciente presentaba clinica de incontinencia urinaria total, con necesidad de usar colector peneano, así como cuadros de pielonefritis de repetición de predominio en el lado derecho. En la cistografía se apreciaba la presencia de una vejiga de contorno festoneado, de baja capacidad con un reflujo vesicoureteral pasivo grado III derecho y activo grado I izquierdo (Fig. 4). Los datos de la cistografía fueron confirmados por el estudio urodinámico.

Se realizó enterocistoplastia de ampliación laparoscópica con un segmento ileal destubularizado de $15 \mathrm{~cm}$. La duración de la cirugía fue de 6 horas. La hemorragia fue de unos 100 cc. El postoperatorio cursó sin incidencias y se procedió al alta hospitalaria al noveno día postoperatorio.

Tras 18 meses de seguimiento el paciente se encuentra continente y no ha tenido nuevos episodios de pielonefritis. La cistografía realizada a los 6 meses de la cirugía a desaparición del reflu-

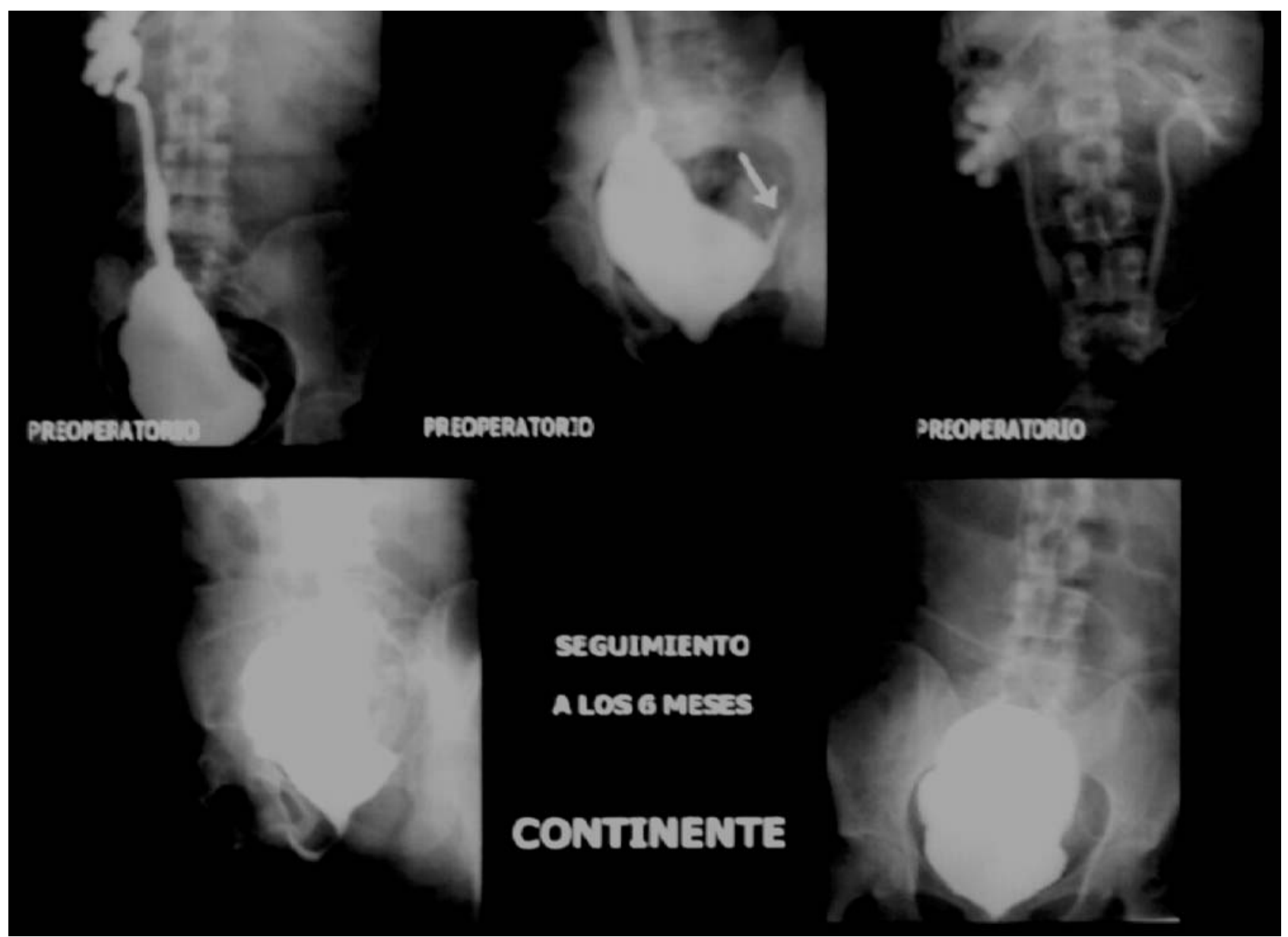

FIGURA 4. Cistografía preoperatoria y a los 6 meses de seguimiento del caso 1. La flecha señala la existencia de reflujo activo izquierdo grado I preoperatorio. 
jo pasivo derecho y activo izquierdo. Sólo se evidenció la persistencia de un reflujo activo grado I derecho.

\section{Caso 2}

Varón de 25 años de edad diagnosticado de vejiga hiperrefléxica secundaria a sección medular traumática desde hacía 3 años. El paciente presentaba disparos miccionales con incontinencia y cuadros de pielonefritis de repetición. En la cistografía se evidenció una vejiga de escasa capacidad, sin reflujo vesicoureteral. En el estudio urodinámico se apreció un disparo miccional desde los 60cc de llenado con altas presiones vesicales que había resultado refractario al tratamiento conservador.

Se realizó enterocistoplastia de ampliación laparoscópica con un segmento ileal de $25 \mathrm{~cm}$ plegado en U. La duración de la cirugía fue de 4,5 horas con un sangrado mínimo. Durante el postoperatorio el paciente presentó un cuadro de constipación, que ya presentaba ocasionalmente antes de la cirugía. Se procedió al alta hospitalaria al duodécimo día postoperatorio.

$\mathrm{El}$ seguimiento ha sido de 6 meses. El paciente se encuentra en programa de autocateterismos, continente y sin nuevos episodios de infección urinaria.

\section{DISCUSIón}

La presencia de una vejiga neurógena de baja compliance obliga a la instauración de un tratamiento con el fin de proteger al tracto urinario superior de la hiperpresión, como primer objetivo, y mejorar la continencia, como segundo objetivo.

Cuando el tratamiento conservador resulta insuficiente es necesario recurrir a tratamientos quirúrgicos, entre los cuales, la enterocistoplastia de ampliación es considerada la técnica de elección ${ }^{1-10,13,14}$, si bien se han descrito complicaciones con esta técnica, tales como fistulas urinarias, litiasis vesical, rotura o perforación y formación de adherencias postoperatorias ${ }^{2,15}$. La existencia de estas posibles complicaciones han llevado a la descripción de técnicas quirúrgicas alternativas como la incisión del detrusor, con o sin miomectomía asociada, que al producir un prolapso de la mucosa y un pseudodivertículo aumenta la capacidad de la vejiga con una reducción asociada de la presión intravesica ${ }^{12,16,17,18}$. A pesar de la aparente sencillez de este método y de los resultados iniciales prometedores ${ }^{17,18}$, la necesidad de realizar enterocistoplastias a medio plazo en algunos pacientes ${ }^{2}$ ha hecho que esta técnica tenga una difusión limitada.

En cuanto a la vía de abordaje, la enterocistoplastia de ampliación suele realizarse mediante laparotomía ${ }^{2-10}$, aunque en los últimos años se han publicado descripciones de procedimientos asistidos por laparoscopia ${ }^{1,12}$ o completamente laparoscópicos ${ }^{11}$.

La cirugia laparoscópica presenta, frente a la cirugía abierta, una clara disminución del dolor postoperatorio, una menor estancia hospitalaria y una menor convalecencia ${ }^{1,19}$. Asimismo, se ha indicado una reducción significativa de la aparición de adherencias intraperitoneales postoperatorias en la cirugía laparoscópica respecto a la cirugía abierta ${ }^{20}$, lo que constituye un factor importante en la elección de la técnica, dado que muchos de los potenciales candidatos pueden ser portadores, como en nuestro primer caso, de una derivación ventrículo-peritoneal o presentar un deterioro de la función renal que en el futuro pudiera requerir diálisis peritoneal.

Desde el punto de vista teórico, la presencia de una derivación ventrículo-peritoneal plantea una serie de problemas. El primero es la posibilidad de infección del catéter de derivación por gérmenes intestinales y el segundo la posibilidad de aumento agudo de la presión intracraneal por la insuflación abdominal. Respecto a la posibilidad de infección del catéter de derivación, ésta es idéntica a la que puede existir en cirugía abierta y se reduce con una adecuada preparación intestinal y profilaxis antibiótica. Respecto al riesgo de aumento de la presión intracraneal, algunos autores han sugerido realizar un estricto control intraoperatorio de la misma mediante monitorización invasiva ${ }^{21,22}$ o mediante Doppler transcraneal $^{23}$, pero la mayoría de los estudios no han demostrado un incremento del riesgo de hipertensión intracraneal en pacientes con derivación ventrículo-peritoneal respecto a los pacientes control. A pesar de esto, es recomendable utilizar presiones de insuflación bajas y vigilar estrechamente la aparición intraoperatoria de hipertensión arterial y bradicardia, que pueden reflejar la presencia de hipertensión intracraneal ${ }^{24-26}$. 
Una desventaja evidente de la utilización de la laparoscopia en la cirugía reconstructiva es, al menos inicialmente, la prolongación del tiempo quirúrgico. Con el fin de reducir la duración de esta técnica quirúrgica se ha propuesto realizar la selección del asa intestinal y la reconstrucción del tránsito mediante minilaparotomía ${ }^{1,12}$. En nuestra opinión, en la enterocistoplastia de ampliación laparoscópica, la prolongación del tiempo quirúrgico no depende de la selección, desfuncionalización y posterior reconstrucción del tránsito por vía laparoscópica.(menos de 60 minutos en nuestra experiencia), sino de la anastomosis vesico-intestinal, por lo que no consideramos justificado perder, al menos parcialmente, las ventajas del acceso laparoscópico en aras de una ligera reducción del tiempo quirúrgico.

El abordaje laparoscópico para la ampliación de vejigas hiperrefléxicas presenta un inconveniente teórico en los casos que tengan asociado un reflujo vesico-ureteral, que consiste en la dificultad técnica de realizar simultáneamente una reimplantación ureteral en un tiempo quirúrgico razonable. En nuestro primer caso, el paciente presentaba un reflujo vesico-ureteral bilateral, pero no consideramos necesario realizar una reimplantación ureteral simultánea, puesto que tras la realización de una enterocistoplastia de ampliación se consigue la resolución espontánea del reflujo vesico-ureteral hasta en un 70-92\% de los casos $^{27-31}$, como así sucedió en nuestro caso.

\section{CONCLUSIONES}

La enterocistoplastia de ampliación laparoscópica constituye una técnica compleja, que precisa una amplia experiencia en cirugía laparoscópica $\mathrm{y}$, sobre todo, en sutura laparoscópica. A pesar de exigir un prolongado tiempo quirúrgico consideramos que se trata de un procedimiento factible que reproduce absolutamente el realizado por cirugía abierta con las ventajas inherentes al abordaje laparoscópico.

\section{REFERENCIAS}

1. Rackley RR, Abdelmalak JB. Laparoscopic augmentation cystoplasty. Surgical technique. Urologic Clinics of North America. 2001;28(3):663-670.

2. Poppas DP, Uzzo RG, Britanisky RG, Mininberg DT. Laparoscopic laser assisted auto-augmentation of the pediatric neurogenic bladder: early experience with urodynamic followup. J Urol. 1996;155(5):1057-1060.
3. Guek ML, Ginsberg DA. Long-term urodynamics followup of bladder augmentation for neurogenic bladder. J Urol. 2003;169(1):195-198.

4. Venn SN, Mundy AR. Long-term results of augmentation cystoplasty. Eur Urol. 1998;34(suppl 1):40-42.

5. Herschorn S, Hewitt RJ. Patient perspective of long-term outcome of augmentation cystoplasty for neurogenic bladder. Urology. 1998;52(4):672-678.

6. Linder A, Leach GE, Raz S. Augmentation cystoplasty in the treatment of neurogenic bladder dysfunction. J Urol. 1983;129(3):491-493.

7. Lockhart JL, Bejany D, Politano VA. Augmentation cystoplasty in the management of neurogenic bladder disease and urinary incontinence. J Urol. 1986;135(5):969-971.

8. Mitchell ME, Kulb TB, Backes DJ. Intestinocystoplasty in combination with clean intermittent catheterization in the management of vesical dysfunction. J Urol. 1986;136(1 Pt2):288-291.

9. Sidi AA, Becher EF, Reddy PK, Dykstra DD. Augmentation enterocystoplasty for the management of voiding dysfunction in spinal cord injury patients. J Urol. 1990;143(1):8385.

10. Andrew MR, Lloyd K. Long-term outcomes of augmentation cystoplasty in spinal cord injured patients. J Urol. 2000;163(suppl 1):52 abstract 226.

11. Elliot SP, Meng MV, Anwar HP, Stoller ML. Complete laparoscopic ileal cystoplasty. Urology. 2002;59(6):939-943.

12. Gill IS, Rackley RR, Meraney AM, Marcello PW, Sung GT. Laparoscopic enterocystoplasty. Urology. 2000;55(2):178181.

13. Hasan ST, Marshall C, Robson WA, Neal DE. Clinical outcome and quality of life following enterocystoplasty for idiopathic detrusor instability and neurogenic bladder dysfunction. Br J Urol. 1995;76(5):551-557.

14. Mundy AR, Stephenson TP. "Clam" ileocystoplasty for the treatment of refractory urge incontinence. $\mathrm{Br} \mathrm{J}$ Urol. 1985;57(6):641-646.

15. Martinez del Castillo ML, Miguelez Lago C, Garcia Merida M, Galiano Duro E, Garcia Soldevila N, Valls Moreno E. Perforación vesical espontánea tras cistoplastia en la infancia. Actas Urol Esp. 2005;9(9):869-878.

16. McNeily, Afshar K, Coleman GU, Johnson HW. Autoaugmentation by detrusor myotomy: its lack of effectiveness in the management of congenital neuropathic bladder. J Urol. 2003;170(4 Pt 2):1643-1646.

17. Siracusano S, Trombetta C, Liguori G, De Giorgi G, d'Aloia G, Di Benedetto P et al. Laparoscopic bladder auto-augmentation in an incomplete traumatic spinal cord injury. Spinal Cord. 2000;38(1):59-61.

18. Kennelly MJ, Gormley EA, McGuire EJ. Early clinical experience with adult bladder auto-augmentation. J Urol. 1994;152(2 Pt1):303-306.

19. Gill IS, Clayman RV, McDougall EM. Advances in urological laparoscopy. J Urol.1995; 154(4):1275-1294.

20. Garrard CL, Clements RH, Nanney L, Davidson JM, Richards WO. Adhesion formation after laparoscopic surgery. Surg Endos. 1999;13(1):10-13.

21. Brown JA, Medlock MD, Dahl DM. Ventriculoperitoneal shunt externalization during laparoscopic prostatectomy. Urology. 2004;63(6):1183-1185.

22. Uzzo RG, Bilsky M, Mininberg DT, Poppas DP. Laparoscopic surgery in children with ventriculoperitoneal shunts: effect of pneumoperitoneum on intracranial pressure.Preliminary experience. Urology. 1997;49(5):753-757. 
23. Ravaoherisoa J, Meyer P, Afriat R, Meyer Y, Sauvanet E, Tricot A et al. Laparoscopic surgery in a patient with ventriculoperitoneal shunt: monitoring of shunt function with transcranial Doppler. Br J Anaesth. 2004;92(3):434-437.

24. Jackman SV, Weingart JD, Kinsman SL, Docimo SG. Laparoscopic surgery in patients with ventriculoperitoneal shunts: safety and monitoring. J Urol. 2000;164(4):13521354 .

25. Al-Mufarrej F, Nolan C, Sookhai S, Broe P. Laparoscopic procedures in adults with ventriculoperitoneal shunts. Surg Laparosc Endosc Percutan Tech. 2005;15(1):28-29.

26. Kimura T, Nakajima K, Wasa M, Yagi M, Kawahara H, Soh $\mathrm{H}$ et al. Succesful laparoscopic fundoplication in children with ventriculoperitoneal shunts. Surg Endosc. 2002;16 (1):215.

27. Lopez-Pereira P, Martinez-Urrutia MJ, Lobato-Romera R, Jaureguizar E. Should we treta vesicoureteral reflux in patients who simultaneously undergo bladder augmentation for neuropathic bladder? J Urol. 2001;165(6 Pt2): 2259-2261.
28. Nasrallah PF, Alibadi HA. Bladder augmentation in patients with neurogenic bladder and vesicoureteral re-flux. J Urol. 1991;146(2 Pt2):563-566.

29. Krishna A, Gough DCS. Evaluation of augmentation cystoplasty in chidhood with reference to vesico-ureteral reflux and urinary infection. Br J Urol. 1994;74(4):465-468.

30. Merlini E, Beseghi U, De Castro R, Perlasca E, Podesta E, Riccipetitoni G. Treatment of vesicoureteral reflux in the neurogenic bladder. Br J Urol. 1993;72(6):969-971.

31. Engel JD, Palmer LS, Cheng EY, Kaplan WE. Surgical versus endoscopic correction of vesicoureteral reflux in children with neurogenic bladder dysfunction. J Urol. 1997; 157(6):2291-2294.

Correspondencia autor: Dr. C. Núñez Mora. Servicio de Urologia. Hospital Univ. La Paz. Po de la Castellana, 261. 28046 Madrid. Tel.: 917277000

E-mail autor: cnunezmora@hotmail.com Información artículo: Original - Cáncer de vejiga (Trabajo aceptado diciembre 2006) 